\title{
Unexpected subspaces of tensor products
}

\author{
Félix Cabello Sánchez, David Pérez-García, and Ignacio Villanueva
}

\begin{abstract}
We describe complemented copies of $\ell_{2}$ both in $C\left(K_{1}\right) \hat{\otimes}_{\pi} C\left(K_{2}\right)$ when at least one of the compact spaces $K_{i}$ is not scattered and in $L_{1}\left(\mu_{1}\right) \hat{\otimes}_{\epsilon} L_{1}\left(\mu_{2}\right)$ when at least one of the measures is not atomic. The corresponding local construction gives uniformly complemented copies of the $\ell_{2}^{n}$ 's in $c_{0} \hat{\otimes}_{\pi} c_{0}$. We continue the study of $c_{0} \hat{\otimes}_{\pi} c_{0}$ showing that it contains a complemented copy of Stegall's space $c_{0}\left(\ell_{2}^{n}\right)$ and proving that $\left(c_{0} \hat{\otimes}_{\pi} c_{0}\right)^{\prime \prime}$ is isomorphic to $\ell_{\infty}\left(\ell_{\infty}^{n} \hat{\otimes}_{\pi} \ell_{\infty}^{n}\right)$, together with other results. In the last section we use Hardy spaces to find an isomorphic copy of $L_{p}$ in the space of compact operators from $L_{q}$ to $L_{r}$, where $1<p, q, r<\infty$ and $1 / r=1 / p+1 / q$.
\end{abstract}

\section{Introduction}

This paper studies subspaces of tensor products of Banach spaces, with emphasis in the 'Varopoulos space' $C\left(K_{1}\right) \hat{\otimes}_{\pi} C\left(K_{2}\right)$. The contents and organization of the article are as follows.

Section 1 contains our main result: $C\left(K_{1}\right) \hat{\otimes}_{\pi} C\left(K_{2}\right)$ has a complemented copy of $\ell_{2}$ as long as $C\left(K_{1}\right)$ is infinite dimensional and $K_{2}$ non-scattered (Theorem 1.2). Actually we give a very explicit representation of $\ell_{2}$, namely, if $\left(f_{n}\right)$ is equivalent to the standard basis of $c_{0}$ in $C\left(K_{1}\right)$ and $\left(g_{n}\right)$ to that of $\ell_{1}$ in $C\left(K_{2}\right)$, then $\left(f_{n} \otimes g_{n}\right)$ spans a complemented copy of $\ell_{2}$ inside $C\left(K_{1}\right) \hat{\otimes}_{\pi} C\left(K_{2}\right)$.

Then we "predualize" Theorem 1.2 to obtain also a complemented copy of $\ell_{2}$ in $L_{1}\left(\mu_{1}\right) \hat{\otimes}_{\epsilon} L_{2}\left(\mu_{2}\right)$ when $\mu_{2}$ is not purely atomic.

Key words and phrases. Tensor product, complemented subspace, Dunford-Pettis property.

Partially supported by BMF 2001-1240 and MTM 2004-02635. 
As an application, we show that the 4-fold tensor product $\hat{\otimes}_{\pi}^{4} c_{0}$ lacks the uniform approximation property, while $\left(\hat{\otimes}_{\pi}^{4} C(K)\right)^{\prime}$ lacks the approximation property if $K$ is non-scattered.

In Section 2 we study the bidual of $c_{0} \hat{\otimes}_{\pi} c_{0}$ and we prove that it is isomorphic to $\ell_{\infty}\left(\ell_{\infty}^{n} \hat{\otimes}_{\pi} \ell_{\infty}^{n}\right)$, but not to $\ell_{\infty} \hat{\otimes}_{\pi} \ell_{\infty}$ which, as we will see, is not a direct factor in any dual Banach space.

Finally, Section 3 deals with injective tensor product of Lebesgue spaces. We use Hankellike operators on the Hardy classes to exhibit a copy of $L_{p}$ in the space of compact operators $\mathfrak{K}\left(L_{q}, L_{r}\right)$ if we are given $1<p, q, r<\infty$ such that $1 / p+1 / q=1 / r$. Complementation, however, will not follow.

Although we have presented our main results without any mention to the Dunford-Pettis property (DPP for short), our research was motivated by the study of the DPP in tensor products.

Recall that a Banach space is said to have the DPP if every weakly compact operator defined on it is completely continuous. (An operator is called completely continuous if it takes weakly Cauchy sequences to norm convergent sequences, and weakly compact if it sends the unit ball into a set with weakly compact closure.) It is a consequence of the work of Dunford and Pettis that $L_{1}(\mu)$ spaces have the DPP, and later Grothendieck, who first isolated this property and named it, proved that $C(K)$ spaces also have it. It is well known that the DPP is stable by complemented subspaces, that reflexive (infinite dimensional) spaces never have the DPP and that, if $X^{\prime}$ has the DPP, so does $X$. The converse is not true, as shown in [20]. It is interesting to remark that Stegall actually constructs a Banach space $X$ such that $X^{\prime}$ has the DPP but $X^{\prime \prime}$ does not: $X=c_{0}\left(\ell_{2}^{n}\right)$ has the DPP because $X^{\prime}=\ell_{1}\left(\ell_{2}^{n}\right)$ has even the Schur property (weakly convergent sequences converge in norm); $X^{\prime \prime}=\ell_{\infty}\left(\ell_{2}^{n}\right)$ lacks the DPP because it contains a complemented copy of $\ell_{2}$.

This example was essentially unique until recently it was proved that $c_{0} \hat{\otimes}_{\pi} c_{0}$ has the same behaviour. The space $c_{0} \hat{\otimes}_{\pi} c_{0}$ has the DPP because its dual $\ell_{1} \hat{\otimes}_{\epsilon} \ell_{1}$ has the Schur property, as in Stegall example. On the other hand Fernando Bombal and the third named author showed that $C\left(K_{1}\right) \hat{\otimes}_{\pi} C\left(K_{2}\right)$ does not have the DPP whenever at least one of the compact spaces $K_{i}$ is not scattered. They proved this showing an explicit example of an operator 
$\varpi: C\left(K_{1}\right) \hat{\otimes}_{\pi} C\left(K_{2}\right) \longrightarrow \ell_{2}$ which is not completely continuous. Soon afterwards, the authors of [10] pushed the same ideas further to exhibit several instances of both projective and injective tensor products of Banach spaces lacking the DPP. In particular, they proved that $\left(c_{0} \hat{\otimes}_{\pi} c_{0}\right)^{\prime \prime}$ lacks the DPP by extending $\varpi: \ell_{\infty} \hat{\otimes}_{\pi} \ell_{\infty} \longrightarrow \ell_{2}$ to an operator $\left(c_{0} \hat{\otimes}_{\pi} c_{0}\right)^{\prime \prime} \longrightarrow \ell_{2}$. Actually, every weakly compact operator on $\ell_{\infty} \hat{\otimes}_{\pi} \ell_{\infty}$ extends to $\left(c_{0} \hat{\otimes}_{\pi} c_{0}\right)^{\prime \prime}$ as the former space is a locally complemented subspace of the latter, see [4]. This provided the essentially second example known to the authors of a Banach space with the DPP whose second dual lacks it. The starting point of our research was to find out whether there is some relation between Stegall's example and $c_{0} \hat{\otimes}_{\pi} c_{0}$. Our main result implies that the above mentioned operator $\varpi: C\left(K_{1}\right) \hat{\otimes}_{\pi} C\left(K_{2}\right) \longrightarrow \ell_{2}$ is in fact a projection, while the corresponding local argument and the results of Section 2 imply that $c_{0} \hat{\otimes}_{\pi} c_{0}$ contains a complemented copy of $c_{0}\left(\ell_{2}^{n}\right)$. Thus, essentially and as far as we know, Stegall's example remains as the sole available example of a Banach space having the DPP and whose bidual lacks it. To tell the truth, we should mention that the space $T$ constructed by Talagrand in $[23]$ has the DPP, its dual has the Schur property, and $T^{\prime \prime}$ lacks the DPP, as it is shown by Núnez in $[\mathbf{1 7}]$. However a close inspection to $T$ reveals that it contains a complemented copy of Stegall's $c_{0}\left(\ell_{2}^{n}\right)$, too.

Notations. The notations and terminology used along the paper will be the standard in Banach space theory, as for instance in [9]. We have written $X \hat{\otimes}_{\pi} Y$ for the projective tensor product of two given Banach spaces $X$ and $Y$, while the injective tensor product is denoted $X \hat{\otimes}_{\epsilon} Y$.

If $Y$ is a complemented subspace of $X$, then the (relative) projection constant of $Y$ in $X$ is the infimum of the norms of all projections from $X$ onto $Y$ and it is denoted $\lambda(Y, X)$.

If $X$ and $Y$ are isomorphic, then the (multiplicative) Banach-Mazur distance between $X$ and $Y$ is

$$
d(X, Y)=\inf \left\{\|T\|\left\|T^{-1}\right\| \text { with } T: X \longrightarrow Y \text { an isomorphism }\right\} .
$$

The topological dual of $X$ is denoted $X^{\prime}$; the value of $x^{\prime} \in X$ at $x \in X$ is often denoted $\left\langle x^{\prime}, x\right\rangle$. If $T: X \longrightarrow Y$ is a (linear, bounded) operator, then the adjoint is denoted by $T^{\prime}$. We 
use $K$ (with or without subscripts) for a compact (Hausdorff) space, while $C(K)$ stands for the space of all continuous functions on $K$ (with values in the ground field), endowed with the supremum norm.

Further notation will be introduced when needed.

\section{Complemented copies of $\ell_{2}$ in $c_{0} \hat{\otimes}_{\pi} L_{\infty}$ and $\ell_{1} \hat{\otimes}_{\epsilon} L_{1}$}

In this Section we show that $\ell_{2}$ lives complemented in $c_{0} \hat{\otimes}_{\pi} C(K)$ if (and only if) $K$ is not scattered. We use well known weak summability arguments to show that certain operators are bounded, the proof ultimately relying on Grothendieck's theorem (or on Orlicz's theorem).

Let $1 \leq p \leq \infty$. A sequence $\left(x_{n}\right)$ is weakly $p$-summable in $X$ if, for every $x^{\prime} \in X^{\prime}$, the scalar sequence $\left(\left\langle x^{\prime}, x_{n}\right\rangle\right)$ is in $\ell_{p}$. In that case we define its $p$-weak norm by

$$
\left\|\left(x_{n}\right)\right\|_{p}^{\omega}=\sup _{\left\|x^{\prime}\right\| \leq 1}\left(\sum_{n}\left|\left\langle x^{\prime}, x_{n}\right\rangle\right|^{p}\right)^{1 / p} .
$$

It is clear (and easy to prove) that $\left(x_{n}\right)$ is weakly $p$-summable if and only if the operator $\ell_{p^{\prime}} \longrightarrow X$ sending $e_{n}$ to $x_{n}$ is bounded (here $p^{\prime}$ denotes the conjugate of $p$, that is, $1 / p^{\prime}+1 / p=1$; if $p=1$ then $c_{0}$ should replace $\left.\ell_{\infty}\right)$. In fact the norm of that operator equals $\left\|\left(x_{n}\right)\right\|_{p}^{\omega}$.

An operator $T: X \longrightarrow Y$ is $p$-summing when it takes weakly $p$-summable sequences into $p$-summable sequences. In that case we have an estimate

$$
\left(\sum_{n=1}^{\infty}\left\|T\left(x_{n}\right)\right\|^{p}\right)^{1 / p} \leq K\left\|\left(x_{n}\right)\right\|_{p}^{\omega},
$$

for some constant $K$ independent on $x_{n}$. The least possible constant in the preceding inequality is denoted $\pi_{p}(T)$. All this can be seen in [9, Chapter 2].

We start with the following simple technical Lemma.

LEMMA 1.1. Let $\left(f_{n}\right)$ be a weakly 2-summable sequence in $C\left(K_{1}\right)$ and $\left(g_{n}\right)$ a bounded sequence in $C\left(K_{2}\right)$. Then $\left(f_{n} \otimes g_{n}\right)$ is weakly 2-summable in $C\left(K_{1}\right) \hat{\otimes}_{\pi} C\left(K_{2}\right)$.

Proof. We may assume $\left\|g_{n}\right\| \leq 1$ for all $n$ and $\left\|\left(f_{n}\right)\right\|_{2}^{\omega} \leq 1$. Let us compute the 2weak norm of the sequence $\left(f_{n} \otimes g_{n}\right)$ in $C\left(K_{1}\right) \hat{\otimes}_{\pi} C\left(K_{2}\right)$. Let $B$ be a norm one functional on 
$C\left(K_{1}\right) \hat{\otimes}_{\pi} C\left(K_{2}\right)$ and let $T: C\left(K_{1}\right) \longrightarrow C\left(K_{2}\right)$ be the associated operator, so that

$$
\langle B, f \otimes g\rangle=\langle T f, g\rangle \quad\left(f \in C\left(K_{1}\right), g \in C\left(K_{2}\right)\right) .
$$

By Grothendieck's inequality $T$ is 2-summing, with $\pi_{2}(T) \leq K_{G}$, where $K_{G}$ is the Grothendieck constant; see [9, theorem 3.5]. Therefore

$$
\left(\sum_{n}\left|\left\langle B, f_{n} \otimes g_{n}\right\rangle\right|^{2}\right)^{1 / 2}\left(\sum_{n}\left|\left\langle T f_{n}, g_{n}\right\rangle\right|^{2}\right)^{1 / 2} \leq\left(\sum_{n}\left\|T f_{n}\right\|^{2}\right)^{1 / 2} \leq \pi_{2}(T)\left\|\left(f_{n}\right)_{n}\right\|_{2}^{w} \leq K_{G} .
$$

Hence $\left\|\left(f_{n} \otimes g_{n}\right)\right\|_{2}^{\omega} \leq K_{G}$.

Our first result follows suit.

THEOREM 1.2. Let $K_{2}$ be a non scattered compact space and $K_{1}$ an infinite compact space. Then $C\left(K_{1}\right) \hat{\otimes}_{\pi} C\left(K_{2}\right)$ has a complemented copy of $\ell_{2}$.

Proof. First of all, let us fix an isomorphic embedding $\jmath: c_{0} \longrightarrow C\left(K_{1}\right)$. Such a $\jmath$ clearly exists: just map the unit basis of $c_{0}$ into a normalized sequence of functions in $C\left(K_{1}\right)$ having disjoint supports. Let $\kappa: C\left(K_{1}\right) \longrightarrow \ell_{\infty}$ be any extension of the inclusion map $c_{0} \longrightarrow \ell_{\infty}$ through $y$ : this can be obtained applying the Hahn-Banach theorem to each coordinate.

Also, since $K_{2}$ is not scattered, there is an operator $\varphi: C\left(K_{2}\right) \longrightarrow \ell_{2}$ mapping a bounded sequence onto the usual basis of $\ell_{2}$. Indeed, $C\left(K_{2}\right)$ contains an isomorphic copy of $\ell_{1}$, and therefore there exists even a surjective operator $C\left(K_{2}\right) \longrightarrow \ell_{2}$ by [9, Corollary 4.16].

Now, define an operator $\varpi: C\left(K_{1}\right) \hat{\otimes}_{\pi} C\left(K_{2}\right) \longrightarrow \ell_{2}$ by

$$
\varpi(f \otimes g)=\kappa(f) \cdot \varphi(g)
$$

where the product is taken coordinatewise. It is clear that $\varpi$ is well defined and also that $\|\varpi\| \leq\|\kappa\|\|\varphi\|$.

Let us construct a bounded right inverse for $\varpi$. Pick a bounded sequence $\left(g_{n}\right)$ such that $\varphi\left(g_{n}\right)=e_{n}$ (in $\left.\ell_{2}\right)$ and let $\left(f_{n}\right)$ be the image under $\jmath$ of the unit basis of $c_{0}$. By Lemma 1.1 the sequence $\left(f_{n} \otimes g_{n}\right)$ is weakly 2-summable in $C\left(K_{1}\right) \hat{\otimes}_{\pi} C\left(K_{2}\right)$ and so we can define a bounded 
operator $\sigma: \ell_{2} \longrightarrow C\left(K_{1}\right) \hat{\otimes}_{\pi} C\left(K_{2}\right)$ taking $\sigma\left(e_{n}\right)=f_{n} \otimes g_{n}$. Moreover

$$
\varpi\left(\sigma\left(e_{n}\right)\right)=\varpi\left(f_{n} \otimes g_{n}\right)=\kappa\left(f_{n}\right) \cdot \varphi\left(g_{n}\right)=e_{n},
$$

so that $\varpi \circ \sigma=\mathbf{1}_{\ell_{2}}$. This shows that $\sigma \circ \varpi$ is a projection on $C\left(K_{1}\right) \hat{\otimes}_{\pi} C\left(K_{2}\right)$ whose range is isomorphic to $\ell_{2}$-and also that $\varpi$ is onto, even if $\varphi$ is not.

In particular $c_{0} \hat{\otimes}_{\pi} \ell_{\infty}$ and $c_{0} \hat{\otimes}_{\pi} C[0,1]$ do contain $\ell_{2}$ as a complemented space. Notice that if both $K_{1}$ and $K_{2}$ are scattered, then $C\left(K_{1}\right) \hat{\otimes}_{\pi} C\left(K_{2}\right)$ has the Dunford-Pettis property (since the dual space is isomorphic to $\ell_{1}\left(K_{1}\right) \hat{\otimes}_{\epsilon} \ell_{1}\left(K_{2}\right)$ which has the Schur property [14]) and it cannot contain a complemented reflexive subspace.

It is clear from the proof of Theorem 1.2 that the complemented copy of $\ell_{2}$ in $C\left(K_{1}\right) \hat{\otimes}_{\pi} C\left(K_{2}\right)$ is just the subspace spanned by the sequence $\left(f_{n} \otimes g_{n}\right)$. Denote it by $\mathfrak{H}$. Since $\varpi \circ \sigma=\mathbf{1}_{\ell_{2}}$ we see that the Banach-Mazur distance between $\mathfrak{H}$ and $\ell_{2}$ is at most $\|\varpi\|\|\sigma\|$. Since $\sigma \circ \varpi$ is a projection onto $\mathfrak{H}$ we see that the relative projection constant of $\mathfrak{H}$ in $C\left(K_{1}\right) \hat{\otimes}_{\pi} C\left(K_{2}\right)$ is bounded by $\|\varpi\|\|\sigma\|$, too. If $\jmath$ is an isometric embedding then $\|\varpi\|=\|\varphi\|$, while (in view of the proof of Lemma 1.1) $\|\sigma\| \leq K_{G} M$, with $M=\sup _{n}\left\|g_{n}\right\|_{C\left(K_{2}\right)}$.

Let us consider a concrete example in detail. For the sake of clarity the action takes place in the Cantor group $\Delta=\{1,-1\}^{\mathbb{N}}$ furnished with the product topology and Haar measure. It is convenient to regard the elements of $\Delta$ as functions $t: \mathbb{N} \longrightarrow\{1,-1\}$. In this setting the Rademacher funtions are just evaluations:

$$
r_{n}(t)=t(n) \quad(n \in \mathbb{N}, u \in \Delta) .
$$

Clearly, $r_{n}$ are in $C(\Delta)$ and so in $L_{p}(\Delta)$ for all $p$. Consider the operator $\rho: \ell_{2} \longrightarrow L_{1}(\Delta)$ defined by $\rho\left(e_{n}\right)=r_{n}$. It is clear that $\|\rho\|=1$ :

$$
\|\rho(x)\|_{L_{2}(\Delta)}=\|x\|_{\ell_{2}} \quad \text { and } \quad\|\rho(x)\|_{L_{1}(\Delta)} \leq\|\rho(x)\|_{L_{2}(\Delta)}
$$

Actually $\rho$ is an isomorphic embedding, according to Khinchin inequality (see $[\mathbf{1 5}]$ or $[\mathbf{9}$, p. 227]) but we will not use this fact. The adjoint $\rho^{\prime}: L_{\infty}(\Delta) \longrightarrow \ell_{2}$ (which is a quotient map) is 
given by

$$
\left\langle\rho^{\prime}(f), e_{n}\right\rangle=\int_{\Delta} r_{n}(t) f(t) d t \quad\left(f \in L_{\infty}(\Delta)\right) .
$$

But the sequence $\left(r_{n}\right)$ is orthonormal in $L_{2}(\Delta)$ and so $\rho^{\prime}\left(r_{n}\right)=e_{n}$. Hence the sequence $\left(e_{n} \otimes r_{n}\right)$ spans a subspace $\mathfrak{H}$ isomorphic to $\ell_{2}$ and complemented in $c_{0} \hat{\otimes}_{\pi} L_{\infty}(\Delta)$. Actually $\mathfrak{H}$ is even a (necessarily complemented) subspace of the smaller space $c_{0} \hat{\otimes}_{\pi} C(\Delta)$. In this case both $d\left(\mathfrak{H}, \ell_{2}\right)$ and $\lambda\left(\mathfrak{H}, c_{0} \hat{\otimes}_{\pi} L_{\infty}(\Delta)\right)$ are bounded by $K_{G}$. See $\left[\mathbf{9}\right.$, p. 29] for numerical bounds of $K_{G}$.

The same ideas can be used to obtain the following local version of Theorem 1.2.

Corollary 1.3. For every $n$, there is $\mathfrak{H}_{n} \subset \ell_{\infty}^{n} \hat{\otimes}_{\pi} \ell_{\infty}^{2^{n}}$ such that $d\left(\mathfrak{H}_{n}, \ell_{2}^{n}\right) \leq K_{G}$ and $\lambda\left(\mathfrak{H}_{n}, \ell_{\infty}^{n} \hat{\otimes}_{\pi} \ell_{\infty}^{2^{n}}\right) \leq K_{G}$

Proof. Let $\Delta^{(n)}=\{1,-1\}^{n}$. We embed $\ell_{2}^{n}$ into $L_{1}\left(\Delta^{(n)}\right)$ using the $n$ Rademachers at our disposal. Then $\mathfrak{H}_{n}$ is the subspace spanned in $\ell_{\infty}^{n} \hat{\otimes}_{\pi} L_{\infty}\left(\Delta^{(n)}\right)$ by the system $\left(e_{k} \otimes r_{k}\right)$ for $1 \leq k \leq n$. Of course $L_{\infty}\left(\Delta^{(n)}\right)$ is isometric to $\ell_{\infty}^{2^{n}}$.

Now, we state and prove a dual version of Theorem 1.2. Consider again the operator $\varpi: c_{0} \hat{\otimes}_{\pi} L_{\infty}(\Delta) \longrightarrow \ell_{2}$ given by

$$
\varpi(f \otimes g)=f \cdot \rho^{\prime}(g) \quad\left(f \in c_{0}, g \in L_{\infty}(\Delta)\right)
$$

Identifying $\ell_{2}$ with its own dual let us consider the adjoint operator

$$
\varpi^{\prime}: \ell_{2} \longrightarrow\left(c_{0} \hat{\otimes}_{\pi} L_{\infty}(\Delta)\right)^{\prime}
$$

It is obvious that $\mathfrak{G}=\varpi^{\prime}\left(\ell_{2}\right)$ is $K_{G}$-isomorphic to $\ell_{2}$ and also that it is $K_{G^{-}}$-complemented in $\left(c_{0} \hat{\otimes}_{\pi} L_{\infty}(\Delta)\right)^{\prime}$. But actually $\varpi^{\prime}$ takes values in $\ell_{1} \hat{\otimes}_{\epsilon} L_{1}(\Delta)$, which is a subspace of $\left(c_{0} \hat{\otimes}_{\pi} L_{\infty}(\Delta)\right)^{\prime}$. Indeed we have $\varpi^{\prime}\left(e_{n}\right)=e_{n} \otimes r_{n} \in \ell_{1} \hat{\otimes}_{\epsilon} L_{1}(\Delta)$ since

$$
\left\langle\varpi^{\prime}\left(e_{n}\right), f \otimes g\right\rangle=\left\langle e_{n}, f \cdot \rho^{\prime}(g)\right\rangle=f(n)\left\langle e_{n}, \rho^{\prime}(g)\right\rangle=\left\langle e_{n}, f\right\rangle\left\langle r_{n}, g\right\rangle=\left\langle e_{n} \otimes r_{n}, f \otimes g\right\rangle
$$

for $f \in c_{0}$ and $g \in L_{\infty}(\Delta)$. Therefore $\mathfrak{G}$ is a complemented subspace of $\ell_{1} \hat{\otimes}_{\epsilon} L_{1}(\Delta)$.

Corollary 1.4. Let $\mu_{1}$ and $\mu_{2}$ be two measures. If $L_{1}\left(\mu_{1}\right)$ is infinite-dimensional and $\mu_{2}$ is not purely atomic, then $L_{1}\left(\mu_{1}\right) \hat{\otimes}_{\epsilon} L_{1}\left(\mu_{2}\right)$ contains a complemented copy of $\ell_{2}$. 
ProOF. The hypotheses imply that $L_{1}\left(\mu_{1}\right)$ contains a complemented copy of $\ell_{1}$ and $L_{1}\left(\mu_{2}\right)$ contains a complemented copy of $L_{1}(\Delta)$. Hence $L_{1}\left(\mu_{1}\right) \hat{\otimes}_{\epsilon} L_{1}\left(\mu_{2}\right)$ contains $\ell_{1} \hat{\otimes}_{\epsilon} L_{1}(\Delta)$ (hence $\left.\ell_{2}\right)$ complemented.

Our next result is a 'formal' consequence of Theorem 1.2 thanks to the main result of [4]. We need the notion of a locally complemented subspace. Suppose $Y$ is a closed subspace of $X$. We say that $Y$ is locally complemented in $X$ if for each finite-dimensional subspace $E \subset X$ there exists an operator $P: E \rightarrow Y$ such that $P$ is the identity on $Y \cap E$, with $\|P\| \leq M$ for some $M$ independent on $E$. A standard compactness argument shows that for dual spaces 'locally complemented' implies 'complemented'. The same is true for Banach spaces which are isomorphic to complemented subspaces of a conjugate space.

Corollary 1.5. Let $K_{1}$ and $K_{2}$ be infinite compact spaces. Then $\left(C\left(K_{1}\right) \hat{\otimes}_{\pi} C\left(K_{2}\right)\right)^{\prime \prime}$ contains a complemented copy of $\ell_{2}$.

ProOF. The main result of $[4]$ states that $C\left(K_{1}\right)^{\prime \prime} \hat{\otimes}_{\pi} C\left(K_{2}\right)^{\prime \prime}$ is a locally complemented subspace of $\left(C\left(K_{1}\right) \hat{\otimes}_{\pi} C\left(K_{2}\right)\right)^{\prime \prime}$. Since 'to be a locally complemented subspace of' is a transitive property and $\ell_{2}$ is reflexive (hence a dual space), it suffices to find a complemented copy of $\ell_{2}$ in $C\left(K_{1}\right)^{\prime \prime} \hat{\otimes}_{\pi} C\left(K_{2}\right)^{\prime \prime}$. But the last space obviously contains a complemented copy of $\ell_{\infty} \hat{\otimes}_{\pi} \ell_{\infty}$, and Theorem 1.2 applies.

REMARK 1. There is an alternate way to Theorem 1.2 which gives better estimates (though less weakly 2-summable sequences). Just use the following result instead of Lemma 1.1.

LEMMA 1.6. Let $\left(f_{n}\right)$ be a weakly 1-summable sequence in $C\left(K_{1}\right)$ and $\left(g_{n}\right)$ a bounded sequence in $C\left(K_{2}\right)$. Then $\left(f_{n} \otimes g_{n}\right)$ is weakly 2-summable in $C\left(K_{1}\right) \hat{\otimes}_{\pi} C\left(K_{2}\right)$.

Proof. The proof is as that of Lemma 1.1, but we use Orlicz theorem (instead of Grothendieck inequality) as stated in [24, theorem 11.11]: every weakly 1 -summable sequence in $L_{1}(\mu)$ is strongly 2-summable, with

$$
\left(\sum_{n}\left\|h_{n}\right\|^{2}\right)^{1 / 2} \leq K_{O}\left\|\left(h_{n}\right)\right\|_{1}^{\omega},
$$


where $K_{O} \leq K_{G}$ and $K_{O}=\sqrt{2}$ in the real case. Hence, if $\left\|g_{n}\right\| \leq 1$ for all $n$ and $\left\|\left(f_{n}\right)\right\|_{1}^{\omega} \leq 1$, we obtain $\left\|\left(f_{n} \otimes g_{n}\right)\right\|_{2}^{\omega} \leq \sqrt{2}$, taking into accout that $C\left(K_{2}\right)^{\prime}=L_{1}(\mu)$ for some measure $\mu$.

REMARK 2. There are two long time open questions related to the results of this Section. On one hand, it is not known whether $c_{0} \hat{\otimes}_{\pi} c_{0}$ has the uniform approximation property (UAP for short), where a Banach space $X$ has UAP if there is a constant $K$ and a function $f: \mathbb{N} \longrightarrow \mathbb{N}$ such that, given $E \subset X$ with $\operatorname{dim} E=k$ there is $T \in \mathfrak{L}(X)$ such that $T$ is the identity on $E$, with $\|T\| \leq K$ and $\operatorname{dim} T(X) \leq f(k)([\mathbf{1 1}, \mathbf{6}])$.

On the other hand, it is also not known whether $c_{0} \hat{\otimes}_{\pi} c_{0}$ is isomorphic to $c_{0} \hat{\otimes}_{\pi} c_{0} \hat{\otimes}_{\pi} c_{0}=\hat{\otimes}_{\pi}^{3} c_{0}$. We first heard of the first problem from Aleksander Pełczyński, and of the second one from Joe Diestel. Note that it follows from the associativity of the projective tensor product that if $c_{0} \hat{\otimes}_{\pi} c_{0}$ is isomorphic to $\hat{\otimes}_{\pi}^{3} c_{0}$, then it is also isomorphic to $\hat{\otimes}_{\pi}^{4} c_{0}$. In this context the following consequence of our results maybe useful.

Corollary 1.7. The space $\hat{\otimes}_{\pi}^{4} c_{0}$ does not have the UAP.

Proof. It follows from Theorem 1.3 that $\hat{\otimes}_{\pi}^{4} c_{0}$ contains uniformly complemented copies of the trace class spaces $\mathfrak{S}_{1}^{n}=\ell_{2}^{n} \hat{\otimes}_{\pi} \ell_{2}^{n}$, and now, using the results in $[\mathbf{2 2}]$, it follows that $\hat{\otimes}_{\pi}^{4} c_{0}$ does not have the UAP.

With a very similar reasoning we have

Corollary 1.8. If $K$ is non-scattered, then $\left(\hat{\otimes}_{\pi}^{4} C(K)\right)^{\prime}$ lacks the AP.

Proof. By Theorem 1.2, $\hat{\otimes}_{\pi}^{4} C(K)$ has a complemented copy of $\ell_{2} \hat{\otimes}_{\pi} \ell_{2}$, therefore $\left(\hat{\otimes}_{\pi}^{4} C(K)\right)^{\prime}$ has a complemented copy of $\left(\ell_{2} \hat{\otimes}_{\pi} \ell_{2}\right)^{\prime}=\mathfrak{L}\left(\ell_{2}\right)$, and this space does not have the AP.

Essentially the same questions as for the $c_{0}$ case are, as far as we know, open for $\ell_{\infty}$ : we do not know whether $\ell_{\infty} \hat{\otimes}_{\pi} \ell_{\infty}$ is isomorphic to $\ell_{\infty} \hat{\otimes}_{\pi} \ell_{\infty} \hat{\otimes}_{\pi} \ell_{\infty}$, and we do not know whether $\left(\ell_{\infty} \hat{\otimes}_{\pi} \ell_{\infty}\right)^{\prime}$ has the AP. 


\section{A description of the bidual of $c_{0} \hat{\otimes}_{\pi} c_{0}$}

In this Section we compare the spaces $\left(c_{0} \hat{\otimes}_{\pi} c_{0}\right)^{\prime \prime}, \ell_{\infty}\left(\ell_{\infty}^{n} \hat{\otimes}_{\pi} \ell_{\infty}^{n}\right)$ and $\ell_{\infty} \hat{\otimes}_{\pi} \ell_{\infty}$. Given a sequence space $S$ and $A \subset \mathbb{N}$, we put

$$
S[A]=\{x \in S: x(n)=0 \text { for } n \notin A\} .
$$

The following result generalizes [3, lemma 2]. The observation that one can (and must) use a Riemann integral to simplify the proof is due to Klaus Floret.

Lemma 2.1. Let $X$ and $Y$ have unconditional bases and let $\left(A_{n}\right)$ and $\left(B_{n}\right)$ be two partitions of $\mathbb{N}$. Let $E$ be the smallest closed subspace of $X \hat{\otimes}_{\pi} Y$ containing every $X\left[A_{n}\right] \otimes Y\left[B_{n}\right]$. Then $E$ is complemented in $X \hat{\otimes}_{\pi} Y$.

Proof. We may and do assume the bases 1-unconditional. We have an action of the Cantor group $\Delta$ on $X$ given by

$$
(t \circ x)(k)=t(n) x(k) \quad\left(k \in A_{n}\right) .
$$

Similarly,

$$
(t \circ y)(k)=t(n) y(k) \quad\left(k \in B_{n}\right)
$$

defines an action of $\Delta$ on $Y$. These actions are continuous. In particular, given $z$ in $X$ (respectively, in $Y$ ), the map $u \longmapsto u \circ z$ is continuous from $\Delta$ to $X$ (respectively, to $Y$ ). Thus we can define a bilinear map $B: X \times Y \longrightarrow X \hat{\otimes}_{\pi} Y$ through the (Riemann) integral

$$
B(x, y)=\int_{\Delta}\left(t \circ x \otimes t^{-1} \circ y\right) d t
$$

It is clear that $\|B\| \leq 1$. Let $P$ denote the linearization of $B$, so that $P(x \otimes y)=B(x, y)$. We have $\|P\|=\|B\| \leq 1$. It is easily seen that $P$ is a projection of $X \hat{\otimes}_{\pi} Y$ onto $E$. In fact

$$
P\left(e_{i} \otimes e_{j}\right)= \begin{cases}e_{i} \otimes e_{j} & \text { if } e_{i} \otimes e_{j} \in E \\ 0 & \text { otherwise }\end{cases}
$$

This completes the proof.

Proposition 2.2. The space $\left(c_{0} \hat{\otimes}_{\pi} c_{0}\right)^{\prime \prime}$ is isomorphic to $\ell_{\infty}\left(\ell_{\infty}^{n} \hat{\otimes}_{\pi} \ell_{\infty}^{n}\right)$. 
Proof. We prepare the ground for Pełczyński decomposition method by showing that $\ell_{\infty}\left(\ell_{\infty}^{n} \hat{\otimes}_{\pi} \ell_{\infty}^{n}\right)$ embeds as a complemented subspace of $\left(c_{0} \hat{\otimes}_{\pi} c_{0}\right)^{\prime \prime}$.

Let $\left(A_{n}\right)$ and $\left(B_{n}\right)$ be two partitions of $\mathbb{N}$ and let $E$ be the subspace of $c_{0} \hat{\otimes}_{\pi} c_{0}$ defined in Lemma 2.1. We already know that $E$ is the range of a contractive projection on $c_{0} \hat{\otimes}_{\pi} c_{0}$. We claim that $E$ is isometric to $c_{0}\left(c_{0}\left[A_{n}\right] \hat{\otimes}_{\pi} c_{0}\left[B_{n}\right]\right)$. It is completely obvious that the closure of $c_{0}\left[A_{n}\right] \otimes c_{0}\left[B_{n}\right]$ in $c_{0} \hat{\otimes}_{\pi} c_{0}$ is isometric to $c_{0}\left[A_{n}\right] \hat{\otimes}_{\pi} c_{0}\left[B_{n}\right]$, and so we treat the latter space as a subspace of $c_{0} \hat{\otimes}_{\pi} c_{0}$. Now, it suffices to show that, given $u_{n} \in c_{0}\left[A_{n}\right] \hat{\otimes}_{\pi} c_{0}\left[B_{n}\right]$, one has

$$
\left\|u_{1}+\cdots+u_{k}\right\|=\max _{1 \leq n \leq k}\left\|u_{n}\right\|
$$

Put $u=u_{1}+\cdots+u_{k}$. That $\|u\| \geq\left\|u_{n}\right\|$ for $1 \leq n \leq k$ is clear since $u_{n}$ is the image of $u$ under a contractive projection. Hence

$$
\|u\| \geq \max _{1 \leq n \leq k}\left\|u_{n}\right\|
$$

To prove the reversed inequality, let $P^{\prime}$ be the adjoint of the averaging projection described in Lemma 2.1 acting on $\left(c_{0} \hat{\otimes}_{\pi} c_{0}\right)^{\prime}$. Notice that

$$
\left\langle P^{\prime} f, x \otimes y\right\rangle=\int_{\Delta} f\left(t \circ x \otimes t^{-1} \circ y\right) d t
$$

for all $f \in\left(c_{0} \hat{\otimes}_{\pi} c_{0}\right)^{\prime}$ and $x, y \in c_{0}$. Every functional in the range of $P^{\prime}$ vanishes on the kernel of $P$ and, in fact, for $x \in c_{0}\left[A_{n}\right], y \in c_{0}\left[B_{m}\right]$ and $f \in\left(c_{0} \hat{\otimes}_{\pi} c_{0}\right)^{\prime}$ one has

$$
\left\langle P^{\prime} f, x \otimes y\right\rangle=\delta_{n m} f(x \otimes y)
$$

Now, let $f$ be a norm one functional attaining the norm on $u$ and let $\phi=P^{\prime} f$. Clearly $\phi(u)=f(u)$ and so

$$
\|u\|=\phi\left(u_{1}\right)+\cdots+\phi\left(u_{k}\right)
$$

Fix $\varepsilon>0$ and choose $x_{n} \in c_{0}\left[A_{n}\right]$ and $y_{n} \in c_{0}\left[B_{n}\right]$ so that

$$
\left|\phi\left(u_{n}\right)\right|<\phi\left(x_{n} \otimes y_{n}\right)+\frac{\varepsilon}{k} \quad \text { with } \quad\left\|x_{n}\right\|=1 \quad \text { and } \quad\left\|y_{n}\right\|=\left\|u_{n}\right\| \text {. }
$$


This can be done because the norm of a bilinear functional equals the norm of its linearization on the corresponding tensor product. We obtain

$$
\begin{aligned}
\|u\| & \leq\left|\phi\left(u_{1}\right)\right|+\cdots+\left|\phi\left(u_{k}\right)\right| \\
& <\phi\left(x_{1} \otimes y_{1}\right)+\cdots+\phi\left(x_{k} \otimes y_{k}\right)+\varepsilon \\
& =\phi\left(\left(x_{1}+\cdots+x_{k}\right) \otimes\left(y_{1}+\cdots+y_{k}\right)\right)+\varepsilon \\
& \leq\|\phi\|\left\|x_{1}+\cdots+x_{k}\right\|\left\|y_{1}+\cdots+y_{k}\right\|+\varepsilon \\
& =\left\|y_{1}+\cdots+y_{k}\right\|+\varepsilon \\
& =\max _{n}\left\|u_{n}\right\|+\varepsilon,
\end{aligned}
$$

and since $\varepsilon$ is arbitrary we are done.

Taking $A_{n}=B_{n}$ successive intervals consisting of $n$ numbers we conclude that $c_{0}\left(\ell_{\infty}^{n} \hat{\otimes}_{\pi} \ell_{\infty}^{n}\right)$ is isometric to a 1-complemented subspace of $c_{0} \hat{\otimes}_{\pi} c_{0}$ and, passing to the biduals, that $\ell_{\infty}\left(\ell_{\infty}^{n} \hat{\otimes}_{\pi} \ell_{\infty}^{n}\right)$ is isometric to a 1-complemented subspace of $\left(c_{0} \hat{\otimes}_{\pi} c_{0}\right)^{\prime \prime}$.

Next, we show that $\left(c_{0} \hat{\otimes}_{\pi} c_{0}\right)^{\prime \prime}$ is a complemented subspace of $\ell_{\infty}\left(\ell_{\infty}^{n} \hat{\otimes}_{\pi} \ell_{\infty}^{n}\right)$. We regard $\ell_{1} \hat{\otimes}_{\epsilon} \ell_{1}$ as the conjugate of $c_{0} \hat{\otimes}_{\pi} c_{0}$, so that $\left(c_{0} \hat{\otimes}_{\pi} c_{0}\right)^{\prime \prime}$ is the conjugate of $\ell_{1} \hat{\otimes}_{\epsilon} \ell_{1}$. Also, we treat $\ell_{\infty}^{n} \hat{\otimes}_{\pi} \ell_{\infty}^{n}$ as the conjugate of $\ell_{1}^{n} \hat{\otimes}_{\epsilon} \ell_{1}^{n}$; and the latter space as a subspace of $\ell_{1} \hat{\otimes}_{\epsilon} \ell_{1}$. Using these conventions we can define an operator $\kappa:\left(c_{0} \hat{\otimes}_{\pi} c_{0}\right)^{\prime \prime} \longrightarrow \ell_{\infty}\left(\ell_{\infty}^{n} \hat{\otimes}_{\pi} \ell_{\infty}^{n}\right)$ taking

$$
(\kappa(u))_{n}=\left.u\right|_{\ell_{1}^{n} \hat{\otimes}_{\epsilon} \ell_{1}^{n}} \in \ell_{\infty}^{n} \hat{\otimes}_{\pi} \ell_{\infty}^{n}
$$

Quite clearly, $\|\kappa\| \leq 1$. Let us construct a projection of $\ell_{\infty}\left(\ell_{\infty}^{n} \hat{\otimes}_{\pi} \ell_{\infty}^{n}\right)$ onto $\left(c_{0} \hat{\otimes}_{\pi} c_{0}\right)^{\prime \prime}$ through $\kappa$. Let $\mathfrak{V}$ be a nontrivial ultrafilter on $\mathbb{N}$ and define $\pi: \ell_{\infty}\left(\ell_{\infty}^{n} \hat{\otimes}_{\pi} \ell_{\infty}^{n}\right) \longrightarrow\left(c_{0} \hat{\otimes}_{\pi} c_{0}\right)^{\prime \prime}$ as

$$
\pi\left(\left(B_{n}\right)_{n}\right)=\text { weak }^{*}-\lim _{\mathfrak{V}(n)} B_{n}
$$

where $\ell_{\infty}^{n} \hat{\otimes}_{\pi} \ell_{\infty}^{n}$ is treated as a subspace of $c_{0} \hat{\otimes}_{\pi} c_{0}$ (hence of the bidual). We check that $\pi$ is a right inverse for $\kappa$ (incidentally, this will show that $\kappa$ is an isomorphic embedding). One only has to show that

$$
B=\text { weak }^{*}-\lim _{n \rightarrow \infty} B_{n} \quad\left(B \in\left(c_{0} \hat{\otimes}_{\pi} c_{0}\right)^{\prime \prime}\right)
$$


where $B_{n}$ is given by

$$
B_{n}(A)=B\left(P_{n}(A)\right) \quad\left(A \in \ell_{1} \hat{\otimes}_{\epsilon} \ell_{1}\right)
$$

and $P_{n}$ is the obvious projection of $\ell_{1} \hat{\otimes}_{\epsilon} \ell_{1}$ onto $\ell_{1}^{n} \hat{\otimes}_{\epsilon} \ell_{1}^{n}$. But this is clear since $P_{n}(A)$ converges to $A$ strongly in $\ell_{1} \hat{\otimes}_{\epsilon} \ell_{1}$ as $n \rightarrow \infty$.

Thus, each of the spaces $\left(c_{0} \hat{\otimes}_{\pi} c_{0}\right)^{\prime \prime}$ and $\ell_{\infty}\left(\ell_{\infty}^{n} \hat{\otimes}_{\pi} \ell_{\infty}^{n}\right)$ is isomorphic to a complemented subspace of the other and the proof will be complete if we show that $\left(c_{0} \hat{\otimes}_{\pi} c_{0}\right)^{\prime \prime}$ is isomorphic to its $\ell_{\infty}$-Sum (see [25, theorem 24]). It clearly suffices to see that $c_{0} \hat{\otimes}_{\pi} c_{0}$ is isomorphic to $c_{0}\left(c_{0} \hat{\otimes}_{\pi} c_{0}\right)$. That $c_{0} \hat{\otimes}_{\pi} c_{0}$ is isomorphic to its $c_{0}$-sum follows from the most elementary version of Pełczyński's method (that in [18]): both spaces are isomorphic to their squares and, in view of Lemma 2.1 each of them contains a complemented copy of the other.

As a by-product of Corollary 1.3, Lemma 2.1 and the proof of the preceding proposition we obtain:

Corollary 2.3. The space $c_{0} \hat{\otimes}_{\pi} c_{0}$ contains a complemented copy of $c_{0}\left(\ell_{2}^{n}\right)$.

Thus Stegall's example remains as the sole known example of a Banach space having DDP and whose bidual lacks it.

The space $\ell_{\infty} \hat{\otimes}_{\pi} \ell_{\infty}$ occupies, to some extent, an intermediate position between $c_{0} \hat{\otimes}_{\pi} c_{0}$ and its bidual. To be more precise, there are isometries

$$
c_{0} \hat{\otimes}_{\pi} c_{0} \stackrel{\jmath}{\longrightarrow} \ell_{\infty} \hat{\otimes}_{\pi} \ell_{\infty} \stackrel{\kappa}{\longrightarrow}\left(c_{0} \hat{\otimes}_{\pi} c_{0}\right)^{\prime \prime}
$$

so that $\kappa \circ \jmath$ is the inclusion of $c_{0} \hat{\otimes}_{\pi} c_{0}$ in its bidual. Here, $\jmath$ is obtained tensorizing (twice) the inclusion of $c_{0}$ in $\ell_{\infty}$ and $\kappa$ is defined through the Aron-Berner (or Davie-Gamelin) extension in a much more general setting; see [4]. For our current purposes it suffices to identify $\ell_{1} \hat{\otimes}_{\epsilon} \ell_{1}$ with the dual of $c_{0} \hat{\otimes}_{\pi} c_{0}$ : in this way $\left(c_{0} \hat{\otimes}_{\pi} c_{0}\right)^{\prime \prime}$ is the conjugate space of $\ell_{1} \hat{\otimes}_{\epsilon} \ell_{1}$ and $\ell_{\infty} \hat{\otimes}_{\pi} \ell_{\infty}$ is just the space generated by the functionals of the form

$$
x \otimes y \longmapsto\langle f, x\rangle\langle g, y\rangle \quad\left(x, y \in \ell_{1}, f, g \in \ell_{\infty}\right)
$$


while $c_{0} \hat{\otimes}_{\pi} c_{0}$ is obtained with $f, g \in c_{0}$. Under these representations the arrows of (1) are just inclusions.

Another possibility is to identify $\left(c_{0} \hat{\otimes}_{\pi} c_{0}\right)^{\prime \prime}$ with the space of all integral operators $\mathfrak{I}\left(\ell_{1}, \ell_{\infty}\right)$. Then $\ell_{\infty} \hat{\otimes}_{\pi} \ell_{\infty}=\mathfrak{N}\left(\ell_{1}, \ell_{\infty}\right)$, the subspace of nucleal operators.

In view of Proposition 2.2 one may wonder if $\ell_{\infty} \hat{\otimes}_{\pi} \ell_{\infty}$ is isomorphic to $\left(c_{0} \hat{\otimes}_{\pi} c_{0}\right)^{\prime \prime}$, too. The following result answers the last question in the negative. Its proof shows that the results in [4] are, to some extent, optimal.

Proposition 2.4. The space $\ell_{\infty} \hat{\otimes}_{\pi} \ell_{\infty}$ is not complemented in any dual space. In particular it is not isomorphic to $\left(c_{0} \hat{\otimes}_{\pi} c_{0}\right)^{\prime \prime}$.

Proof. Recall that if a Banach space is complemented in some dual space, then it is complemented in every space containing it as a locally complemented subspace.

It is proved in [4] that $\ell_{\infty} \hat{\otimes}_{\pi} \ell_{\infty}$ is locally complemented in $\left(c_{0} \hat{\otimes}_{\pi} c_{0}\right)^{\prime \prime}$. Thus if $\ell_{\infty} \hat{\otimes}_{\pi} \ell_{\infty}$ were complemented in some dual space, it should be complemented in $\left(c_{0} \hat{\otimes}_{\pi} c_{0}\right)^{\prime \prime}$. We complete the proof by showing that this is not the case.

For the remainder of this Section we abbreviate $\ell_{\infty} \hat{\otimes}_{\pi} \ell_{\infty}$ to $\mathfrak{N}$ and $\left(c_{0} \hat{\otimes}_{\pi} c_{0}\right)^{\prime \prime}$ to $\mathfrak{I}$.

Consider the whole exact sequence

$$
0 \longrightarrow \mathfrak{N} \stackrel{\kappa}{\longrightarrow} \mathfrak{I} \stackrel{\pi}{\longrightarrow} \mathfrak{I} / \mathfrak{N} \longrightarrow 0
$$

and recall that in such a diagram the subspace is complemented in the middle space if and only if the quotient map $\pi$ admits a (linear and bounded) section, that is, there is an operator

$$
S: \mathfrak{I} / \mathfrak{N} \longrightarrow \mathfrak{I}
$$

such that $\pi \circ S$ is the identity on $\mathfrak{I} / \mathfrak{N}$. We shall see that such a $S$ cannot exist. Consider the operator $\delta: \ell_{\infty} \longrightarrow \mathfrak{I}$ given by

$$
\delta(f)=\sum_{n=1}^{\infty} f(n)\left(e_{n} \otimes e_{n}\right) \quad\left(f \in \ell_{\infty}\right),
$$

where the summation of the series is performed in the weak* topology of $\mathfrak{I}=\left(\ell_{1} \hat{\otimes}_{\epsilon} \ell_{1}\right)^{\prime}$. It is clear that $\delta$ is an isometric embedding. A moment's reflection shows that $\mathfrak{N} \cap \delta\left(\ell_{\infty}\right)=\delta\left(c_{0}\right)$. 
Thus the composition $\pi \circ \delta$ factorizes through the quotient $\ell_{\infty} / c_{0}$ and we have a commutative diagram

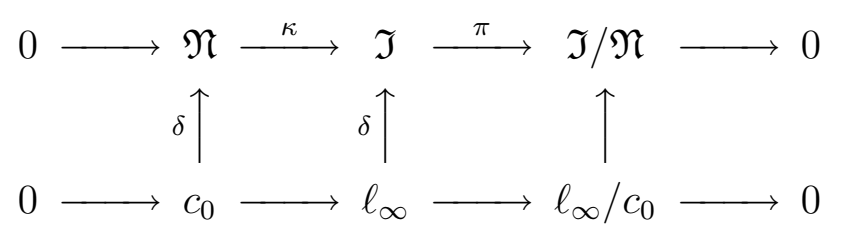

where the rows are exact and the vertical arrows are isometric embeddings. Therefore $\mathfrak{I} / \mathfrak{N}$ contains a subspace isometric to $\ell_{\infty} / c_{0}$. But the latter space contains a further subspace isometric to $c_{0}(\Gamma)$, where $\Gamma$ has the power of continuum. Since there is no one-to-one operator from $c_{0}(\Gamma)$ into the dual of a separable Banach space ( $\operatorname{such}$ as $\ell_{1} \hat{\otimes}_{\epsilon} \ell_{1}$ ) we conclude that $\pi$ cannot have a linear and bounded section.

REMARK 3. There is a number of intriguing questions about $\ell_{\infty} \hat{\otimes}_{\pi} \ell_{\infty}$. For instance, it is not known whether $\ell_{\infty} \hat{\otimes}_{\pi} \ell_{\infty}$ contains a complemented subspace isomorphic to $c_{0}$ or not. Note that Proposition 2.4 leaves the possibility of an affirmative answer open. We know very little about how to embeed $c_{0}$ into $\ell_{\infty} \hat{\otimes}_{\pi} \ell_{\infty}$. It is clear that if $\mathfrak{S}$ is a subspace isomorphic to $c_{0}$ and there is another subspace $\mathfrak{M} \subset \ell_{\infty} \hat{\otimes}_{\pi} \ell_{\infty}$ containing $\mathfrak{S}$ and isomorphic to $\ell_{\infty}$, then $\mathfrak{S}$ is not complemented in $\ell_{\infty} \hat{\otimes}_{\pi} \ell_{\infty}$. On the other hand, we know that $\delta\left(c_{0}\right)$ is uncomplemented in $\ell_{\infty} \hat{\otimes}_{\pi} \ell_{\infty}$ (this follows, e.g., from [5]). However, $\ell_{\infty} \hat{\otimes}_{\pi} \ell_{\infty}$ is very "thin" around the diagonal:

Claim 2.5. No subspace of $\ell_{\infty} \hat{\otimes}_{\pi} \ell_{\infty}$ containing $\delta\left(c_{0}\right)$ is isomorphic to $\ell_{\infty}$.

ProOF. With the same notation as before, suppose $\delta\left(c_{0}\right) \subset \mathfrak{M} \subset \mathfrak{N}$, with $\mathfrak{M}$ isomorphic to $\ell_{\infty}$. Then $\mathfrak{M} / \delta\left(c_{0}\right)$ is isomorphic to a subspace of $\mathfrak{N} / \delta\left(c_{0}\right)$. It is known that there is only one isomorphic embedding of $c_{0}$ into $\ell_{\infty}$, up to automorphisms of $\ell_{\infty}$ (the Lindenstrauss-Rosenthal theorem [16]; there is a remarkable simple proof in $[\mathbf{7}])$. Hence $\mathfrak{M} / \delta\left(c_{0}\right)$ is isomorphic to $\ell_{\infty} / c_{0}$.

On the other hand, applying diamond's lemma (see any book in basic algebra or just chase the diagram below) to $\mathfrak{N}$ and $\delta\left(\ell_{\infty}\right)$ in $\mathfrak{I}$ and, bearing in mind that $\delta\left(\ell_{\infty}\right) \cap \mathfrak{N}=\delta\left(c_{0}\right)$, we get 
the commutative diagram

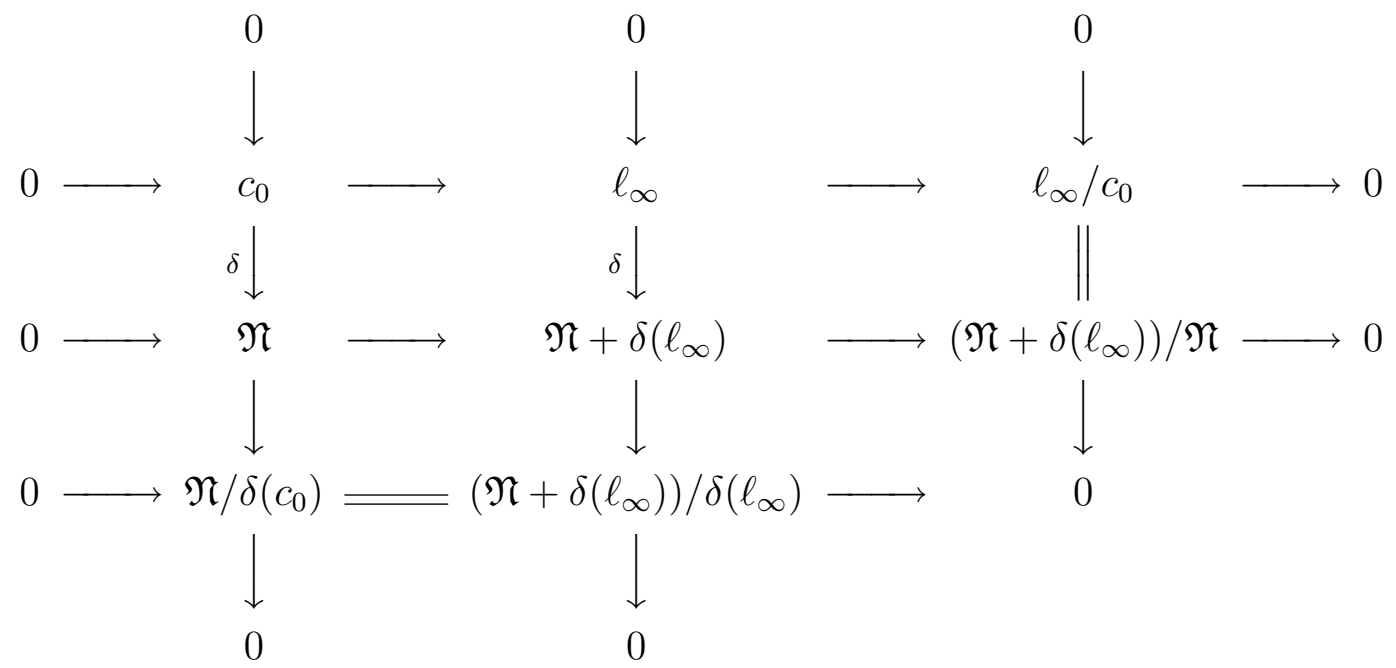

where the rows and columns are exact sequences. But $\ell_{\infty}$ is injective amongst Banach spaces and so the middle vertical sequence splits: this implies that $\mathfrak{N}+\delta\left(\ell_{\infty}\right)$ (hence $\left.\mathfrak{I}\right)$ contains an isomorphic copy of $\mathfrak{N} / \delta\left(c_{0}\right)$ (hence of $\left.\ell_{\infty} / c_{0}\right)$. A contradiction.

REMARK 4. Proposition 2.2 cannot be predualized (twice) to obtain an isomorphism between $c_{0} \hat{\otimes}_{\pi} c_{0}$ and $c_{0}\left(\ell_{\infty}^{n} \hat{\otimes}_{\pi} \ell_{\infty}^{n}\right)$. Actually, the latter space is (as every $c_{0}$-sum of finite dimensional spaces) isomorphic to a subspace of $c_{0}$, while the former is not. This follows from the fact (proved by Stehle in [21]) that there are subspaces of $c_{0} \hat{\otimes}_{\pi} c_{0}$ failing the DPP.

\section{3. $L_{p}$ as a space of compact operators}

This Section has only loose connections with the preceding ones. As a motivation of our closing result, let us consider numbers $p, q$ and $r$ so that $1 / p+1 / q=1 / r$. Hölder's inequality tells us that the bilinear operator

$$
m: L_{p} \times L_{q} \longrightarrow L_{r}
$$

sending $(f, g)$ to the product $f \cdot g$ is continuous: actually $\|m\|=1$. It is easy to see that it is also surjective: indeed, if $h \in L_{r}$, and we write $h=u|h|$ with $u$ unitary, then $f=u|h|^{r / p}$ belongs to $L_{p}, g=|h|^{r / q}$ belongs to $L_{q}$ and $h=f \cdot g$. Moreover, $\|h\|_{r}=\|f\|_{p}\|g\|_{q}$. Thus, the linearization of $m$ is a quotient operator $\tilde{m}: L_{p} \hat{\otimes}_{\pi} L_{q} \longrightarrow L_{r}$. One may wonder if $\tilde{m}$ has a 
right inverse so that there is a complemented copy of $L_{r}$ in $L_{p} \hat{\otimes}_{\pi} L_{q}$-as it is the case for the operator $C\left(K_{1}\right) \hat{\otimes}_{\pi} C\left(K_{2}\right) \longrightarrow \ell_{2}$ appearing in Section 1. All we know is:

- In general $\tilde{m}$ does not have a right inverse. Indeed, take $p=q=2$ so that $r=1$. Then $L_{1}$ cannot be a subspace of $L_{2} \hat{\otimes}_{\pi} L_{2}$ because the latter space has the Radon-Nikodým property (a hereditary property) while the former lacks it.

- However $\tilde{m}$ has a right inverse in the purely atomic case, so that $\ell_{p} \hat{\otimes}_{\pi} \ell_{q}$ has a complemented copy of $\ell_{r}([\mathbf{1}]$ or $[\mathbf{3}$, proposition 1$])$.

¿From now on, we assume $r>1$. The main result of $[\mathbf{3}]$ implies that there is always a local right inverse for $\tilde{m}$ (explicit constructions are also available). As the reader can imagine we say that $P: X \longrightarrow Z$ has a local right inverse if, for each finite-dimensional $E \subset Z$ there is an operator $S: E \longrightarrow X$ such that $P \circ S=\mathbf{1}_{E}$, with $\|S\| \leq M$ for some $M$ independent on $E$.

Thus, if we consider the whole exact sequence

$$
0 \longrightarrow \operatorname{ker} \tilde{m} \longrightarrow L_{p} \hat{\otimes}_{\pi} L_{q} \stackrel{\tilde{m}}{\longrightarrow} L_{r} \longrightarrow 0
$$

we have that ker $\tilde{m}$ is locally complemented in $L_{p} \hat{\otimes}_{\pi} L_{q}$ (see, e.g., [12]). Since our hypotheses imply that $L_{p} \hat{\otimes}_{\pi} L_{q}$ is a dual space, namely the dual of $L_{p^{\prime}} \hat{\otimes}_{\epsilon} L_{q^{\prime}}=\mathfrak{K}\left(L_{p}, L_{q^{\prime}}\right)$, we see that the above sequence splits if and only if ker $\tilde{m}$ is complemented in some dual space. Of course this would be the case if $\tilde{m}$ were weak* continuous. But an operator $T: L_{p} \hat{\otimes}_{\pi} L_{q} \longrightarrow L_{r}$ is weak* continuous if and only if $T^{\prime}: L_{r^{\prime}} \longrightarrow \mathfrak{L}\left(L_{p}, L_{q^{\prime}}\right)$ takes values in $\mathfrak{K}\left(L_{p}, L_{q^{\prime}}\right)$ and $\tilde{m}^{\prime}(f)$ is compact only if $f=0$. At this juncture it is not clear whether $\mathfrak{K}\left(L_{p}, L_{q^{\prime}}\right)$ contains a copy of $L_{r^{\prime}}$ when $1 / p+1 / q=1 / r$. The following result answers this question in the affirmative.

Proposition 3.1. Let $1<p, q, r<\infty$ be such that $1 / p+1 / q=1 / r$. Then $\mathfrak{K}\left(L_{q}, L_{r}\right)$ contains a copy of $L_{p}$.

Notice that we have relabeled the involved parameters. We will prove the analogous statement about the Hardy classes. Recall that the Hardy space $H_{p}=H_{p}(\mathbb{D})$ consists of those 
analytic functions $f: \mathbb{D} \rightarrow \mathbb{C}$ such that

$$
\|f\|_{H_{p}}=\sup _{0<r<1}\left(\frac{1}{2 \pi} \int_{0}^{2 \pi}\left|f\left(r e^{i \theta}\right)\right|^{p} d \theta\right)^{1 / p}<\infty .
$$

The space $H_{p}$ is a Banach space for $1 \leq p<\infty$. The boundary values

$$
f\left(e^{i \theta}\right)=\lim _{r \rightarrow 1} f\left(r e^{i \theta}\right)
$$

exist almost everywhere in $\mathbb{T}$ and $\|f\|_{H_{p}}=\|f\|_{L_{p}(\mathbb{T})}$. This implies that $H_{p}$ is isometric to $H_{p}(\mathbb{T})$, the subspace spanned by the functions $\left\{e^{i n \theta}: n \geq 0\right\}$ in $L_{p}(\mathbb{T})$. This correspondence sends Taylor series into Fourier series: if $f=\sum_{n=0}^{\infty} a_{n} z^{n}$ is the Taylor series of $f \in H_{p}$, then the boundary value of $f$ has Fourier series $\sum_{n=0}^{\infty} a_{n} e^{i n \theta}$. ¿From now on we treat $H_{p}$ as a subspace of $L_{p}(\mathbb{T})$. The map

$$
\sum_{-\infty}^{\infty} a_{k} e^{i k \theta} \longmapsto \sum_{k=0}^{\infty} a_{k} e^{i k \theta}
$$

is often called the Riesz projection. It is bounded on $L_{p}=L_{p}(\mathbb{T})$ if and only if $1<p<\infty$. In this case $H_{p}$ is a complemented subspace of $L_{p}$ and, actually, the two spaces are linearly isomorphic. Let us prove the result.

Proposition 3.2. Let $1<p, q, r<\infty$ satisfy $1 / p+1 / q=1 / r$. Then $H_{p}$ is isomorphic to a closed subspace of $\mathfrak{K}\left(H_{q}, H_{r}\right)$.

Proof. Every $f \in H_{p}$ induces a Hankel-like operator $\mathcal{H}(f): H_{q} \rightarrow H_{r}$ defined by

$$
\mathcal{H}(f)(g)=\mathcal{R}(f \bar{g}) \quad\left(g \in H_{q}\right)
$$

where $\mathcal{R}$ is the Riesz projection on $L_{r}$. We have $\|\mathcal{H}(f)(g)\|_{r} \leq\|\mathcal{R}\|_{\mathfrak{L}\left(L_{r}\right)}\|f\|_{p}\|g\|_{r}$ by Hölder inequality. Hence

$$
\left\|\mathcal{H}(f): H_{q} \rightarrow H_{r}\right\| \leq\|\mathcal{R}\|_{\mathfrak{L}\left(L_{r}\right)}\|f\|_{p}
$$

and so

$$
\left\|\mathcal{H}: H_{p} \rightarrow \mathfrak{L}\left(H_{q}, H_{r}\right)\right\| \leq\|\mathcal{R}\|_{\mathfrak{L}\left(L_{r}\right)} .
$$


Let us see that $\mathcal{H}(f)$ is always compact. Taking $f=z^{k}$ and $g=z^{l}$, we have

$$
\mathcal{H}\left(z^{k}\right)\left(z^{l}\right)=\mathcal{R}\left(z^{k-l}\right)= \begin{cases}z^{k-l} & \text { for } l \leq k \\ 0 & \text { for } l>k .\end{cases}
$$

Thus $\mathcal{H}(f)$ has finite rank when $f=z^{k}$ with $k \in \mathbb{N}$. By linearity $\mathcal{H}(f)$ has finite rank when $f$ is a polynomial and since polynomials are dense in $H_{p}$ and $\mathcal{H}$ is continuous we see that $\mathcal{H}(f)$ is compact for every $f \in H_{p}$.

It remains to verify that $\|\mathcal{H}(f)\| \geq k\|f\|_{p}$ for some constant $k$ independent on $f \in H_{p}$. Take $f \in H_{p}$ with $\|f\|_{p}=1$. Identifying $f$ with its boundary value, there exists $g \in L_{p^{\prime}}$ such that $\|g\|_{p^{\prime}}=1$ and

$$
\langle g \mid f\rangle=\int_{\mathbb{T}} f \bar{g} d \theta=1
$$

But $\langle g \mid f\rangle=\langle\mathcal{R}(g) \mid f\rangle$ and so we can assume that the $g$ appearing in (3) belongs to $H_{p^{\prime}}$, with $\|g\|_{p^{\prime}} \leq\|\mathcal{R}\|_{\mathfrak{L}\left(L_{p^{\prime}}\right)}$. Now since $1 / p^{\prime}=1 / q+1 / r^{\prime}$ there exist a factorization $g=g_{1} g_{2}$, with $g_{1} \in H_{q}, g_{2} \in H_{r^{\prime}}$ and $\|g\|_{p^{\prime}}=\left\|g_{1}\right\|_{q}\left\|g_{2}\right\|_{r^{\prime}}$. Without loss of generality we may assume $\left\|g_{2}\right\|_{r^{\prime}}=1$ and $\left\|g_{1}\right\|_{q}=\|g\|_{p^{\prime}}$. Since

$$
1=\int_{\mathbb{T}} f \cdot \bar{g}_{1} \cdot \bar{g}_{2} d \theta=\int_{\mathbb{T}} \mathcal{R}\left(f \cdot \bar{g}_{1}\right) \cdot \bar{g}_{2} d \theta
$$

we obtain $\left\|\mathcal{H}(f)\left(g_{1}\right)\right\|_{H_{r}}=\left\|\mathcal{R}\left(f \bar{g}_{1}\right)\right\|_{L_{r}} \geq 1$ and thus

$$
\|\mathcal{H}(f)\|_{\mathfrak{K}\left(H_{q}, H_{r}\right)} \geq \frac{1}{\|\mathcal{R}\|_{\mathfrak{L}\left(L_{p^{\prime}}\right)}}\|f\|_{p}
$$

which completes the proof.

Remark 5. A Hankel matrix $A=(A[i, j])_{i, j \geq 0}$ is one such that $A[i, j]=\alpha_{i+j}$ for some sequence $\left(\alpha_{n}\right)$, that is, it has the form

$$
\left[\begin{array}{cccc}
\alpha_{0} & \alpha_{1} & \alpha_{2} & \ldots \\
\alpha_{1} & \alpha_{2} & \ldots & \ldots \\
\alpha_{2} & \ldots & \ldots & \ldots \\
\ldots & \ldots & \ldots & \ldots
\end{array}\right]
$$


It is easily seen that the matrix of $\mathcal{H}(f)$ with respect to the basis $\left(z^{k}\right)_{k \geq 0}$ in $H_{q}$ and $H_{r}$ is a Hankel matrix, with

$$
\mathcal{H}(f)[i, j]=\widehat{f}(i+j)
$$

where $\widehat{f}(n)$ denotes the $n$-th Fourier coefficient of (the boundary value of) $f$. Conversely, if the matrix of a bounded operator $T: H_{q} \rightarrow H_{r}$ with respect to $\left(z^{k}\right)$ satisfies $T[i, j]=\tau_{i+j}$, for some sequence $\tau_{n}$, then

$$
f=\sum_{k=0}^{\infty} \tau_{k} z^{k}
$$

belongs to $H_{p}$ and $T=\mathcal{H}(f)$. We leave the details to the interested reader.

We do not known whether $\mathcal{H}\left(H_{p}\right)$ is complemented in $\mathfrak{K}\left(H_{q}, H_{r}\right)$ when $1<p<\infty$. It should be mentioned that the subspace of Hankel operators is uncomplemented in $\mathfrak{K}\left(H_{2}\right)$, as it follows from a result of Kislyakov [13]. We remark, however, that the Hankel operators in $\mathfrak{K}\left(H_{2}\right)$ form a subspace isomorphic to $C(\mathbb{T}) / A$, where $A$ is the disk algebra (see [19]), and not to the disk algebra itself. This is due to the unboundedness of the Riesz projection on $C(\mathbb{T})$.

\section{References}

[1] A. Arias and J.D. Farmer, On the structure of tensor products of $\ell_{p}$ spaces, Pacific J. Math. 175 (1996) $13-37$.

[2] F. Bombal and I. Villanueva, On the Dunford-Pettis property of the tensor product of $C(K)$ spaces, Proc. Amer. Math. Soc. 129 (2000) 1359-1363.

[3] F. Cabello Sánchez, Complemented subspaces of spaces of multilinear forms and tensor products. J. Math. Anal. Appl. 254 (2001), no. 2, 645-653.

[4] F. Cabello Sánchez and R. García, The bidual of a tensor product of Banach spaces. Rev. Mat. Iberoamericana, to appear.

[5] F. Cabello Sánchez, R. García and I. Villanueva, On a question of Pełczyński about multilinear operators, Bull. Polish Acad. Sci. 48 (4) (2000) 341-345.

[6] P. Casazza, Approximation properties, Handbook on the Geometry of Banach Spaces, Vol. I, W.B. Johnson and J. Lindenstrauss (Editors), Elsevier, Amsterdam, 2001.

[7] J.M.F. Castillo and Y. Moreno, On the Lindenstrauss-Rosenthal theorem, Israel J. Math. 140 (2004) 253270 .

[8] A. Defant and K. Floret, Tensor norms and operator ideals. North-Holland, 1993. 
[9] J. Diestel, H. Jarchow and A. Tonge, Absolutely Summing Operators. Cambridge Univ. Press, 1995.

[10] M. González and J. Gutiérrez, The Dunford-Pettis property on tensor products, Math. Proc. Cambridge Philos. Soc. 131 (2001), 185-192.

[11] S. Heinrich, Ultraproducts in Banach space theory, J. Reine Angew. Math. 313 (1980) 72-104.

[12] W.B. Johnson, Extensions of $c_{0}$, Positivity 1 (1997) 55-74.

[13] S.V. Kislyakov, Projectors onto the set of Hankel matrices. Investigations on linear operators and the theory of functions XII, Zap. Nauch. Sem. Leningrad Otdel. Mat. Inst. Steklov (LOMI) 126 (1983) 109-116.

[14] S. Kwapień and A. Pełczyński, The main triangle projection in matrix spaces and its applications, Studia Math. 34 (1970) 43-68.

[15] R. Latała and K. Oleszkiewicz, On the best constant in the Khinchin-Kahane inequality, Studia Math. 109 (1994) 101-104.

[16] J. Lindenstrauss and H.P. Rosenthal, Automorphisms in $c_{0}, \ell_{1}$ and $m$, Israel J. Math. 9 (1969) $227-239$.

[17] C. Núñez, A conjecture about the Dunford-Pettis property, Portugal. Math. 46 (1989) 31-135.

[18] A. Pełczyński, On the isomorphism of the spaces $m$ and $M$, Bull. Polish Acad. Sci. 6 (11) (1958) 695-696.

[19] V.V. Peller, An excursion into the theory of Hankel operators. Holomorphic spaces (Berkeley 1995) 65-120, Math. Sci. Res. Inst. Publ. 33. Cambrigde University Press, Cambrigde 1998.

[20] C. Stegall, Duals of certain spaces with the Dunford-Pettis property, Notices Amer. Math. Soc. 19 (1972) A-799.

[21] S.P. Stehle, Projective tensor products and the Dunford-Pettis property, Bull. Austral. Math. Soc. 37 (1988) 107-111.

[22] A. Szankowski, On the Uniform Aproximation Property in Banach spaces, Israel J. Math. 49 (1984) 343-359.

[23] M. Talagrand, La propriété de Dunford-Pettis dans $\mathcal{C}(K, E)$ et $L^{1}(E)$, Israel J. Math. 44 (1983) 317-321.

[24] N. Tomczak-Jaegermann, Banach-Mazur Distances and Finite-Dimensional Operator Ideals. Longman Scientific and Technical, 1989.

[25] P. Wojtaszczyk, Banach spaces for analysts. Cambridge Univ. Press, 1991. 
Departamento de Matemáticas, Uex. Avenida de Elvas 06071-Badajoz, España

E-mail address: fcabello@unex.es

Área de Matemática Aplicada, Departamento de Matemáticas y Física Aplicadas y Ciencias de la Naturaleza, Escuela Superior de Ciencias Experimentales y Tecnología, Universidad Rey Juan Carlos, Edificio Departamental II, 28933 Móstoles (Madrid)

E-mail address: david.perez.garcia@urjc.es

Departamento de Análisis Matemático, Facultad de Matemáticas, Universidad Complutense DE MADRID, MAdrid 28040, Spain

E-mail address: ignaciov@mat.ucm.es 